\title{
Time-reversal symmetry breaking by $(d+i d)$ density-wave state in underdoped cuprate superconductors
}

\author{
Sumanta Tewari, Chuanwei Zhang, Victor M. Yakovenko, and S. Das Sarma \\ Condensed Matter Theory Center, Department of Physics, \\ University of Maryland, College Park, Maryland 20742-4111, USA
}

\begin{abstract}
It was proposed that the $i d_{x^{2}-y^{2}}$ density-wave state (DDW) may be responsible for the pseudogap behavior in the underdoped cuprates. Here we show that the admixture of a small $d_{x y}$ component to the DDW state breaks the symmetry between the counter-propagating orbital currents of the DDW state and, thus, violates the macroscopic time-reversal symmetry. This symmetry breaking results in a non-zero polar Kerr effect, which has recently been observed in the pseudogap phase.

PACS numbers: 74.72.-h, 74.25.Nf, 71.27.+a
\end{abstract}

Introduction. The nature of the pseudogap in the cuprate high-temperature superconductors is a much-debated and still unresolved issue [1]. Many of the anomalous properties associated with the pseudogap can be best described as resulting from the order parameters [2], such as those of various density-wave states, competing with the $d$-wave superconductivity. If a density-wave state were to have an energy gap of the $d$-wave symmetry on the Fermi surface, in conformity with the pseudogap, the natural candidate would be the $i d_{x^{2}-y^{2}}$ density wave (DDW) state. Indeed, much of the phenomenology of the cuprates in the underdoped regime can be unified [3, 4, 5] by making a single assumption that the ordered DDW state is responsible for the pseudogap. However, the situation is still controversial, because there is no convincing direct experimental evidence for a phase transition to another state in the underdoped regime, besides the antiferromagnetic order close to half-filling.

The recent observation of quantum oscillations [6] in underdoped YBCO in high magnetic fields has renewed interest in the ordered DDW state [7]. The special coherence factors of the DDW state reduce the hole pockets to appear as Fermi arcs in angle resolved photo emission experiments [8]. An important recent development in the context of the pseudogap is the observation of a non-zero polar Kerr effect (PKE) in the underdoped YBCO [9], which demonstrates the macroscopic time-reversal-symmetry breaking. The PKE appears roughly at the same temperature where the pseudogap develops [9], and, near the optimal doping, appears at a temperature below the superconducting transition temperature. This observation suggests that the time-reversal symmetry breaking and the pseudogap in the cuprates have the same physical origin, which is unrelated to the superconductivity. Similar conclusion was reached earlier, using muon spin relaxation experiments, in Ref. [10]. In this Letter, we examine the PKE of the ordered DDW state, and show that it can give rise to a nonzero signal only if there is an admixture of a $d_{x y}$ component to the total order parameter amplitude. There can be different mechanisms for spontaneous generation of the $d_{x y}$ potential in the mean-field Hamiltonian due to microscopic interactions between electrons [11] or due to a structural transition with the appropriate symmetry. It is important to stress, however, that the amplitude of the $d_{x y}$ admixture, as deduced from the PKE signal [9], is tiny relative to the main $i d_{x^{2}-y^{2}}$ component. Thus, it may be difficult to uncover it unless the experiment directly probes broken time-reversal symmetry. Superconducting order parameters of similar form, explaining experimental observation of broken time-reversal symmetry, have been discussed in [12].

$i d_{x^{2}-y^{2}}$ and $(d+i d)$ density-wave states. The commensurate DDW state [11] is described by the following order parameter, which is a particle-hole singlet in spin space,

$$
\left\langle\hat{c}_{\boldsymbol{k}+\boldsymbol{Q}, \alpha}^{\dagger} \hat{c}_{\boldsymbol{k}, \beta}\right\rangle \propto i W_{\boldsymbol{k}} \delta_{\alpha \beta}, W_{\boldsymbol{k}}=\frac{W_{0}}{2}\left(\cos k_{x}-\cos k_{y}\right) .
$$

Here $\hat{c}^{\dagger}$ and $\hat{c}$ are the electron creation and annihilation operators on the square lattice of the copper atoms, $\boldsymbol{k}=\left(k_{x}, k_{y}\right)$ is the two-dimensional momentum, $\boldsymbol{Q}=(\pi, \pi)$ is the wave vector of the density wave, and $\alpha$ and $\beta$ are the spin indices. Since the order parameter involves $\delta_{\alpha \beta}$, we do all of our calculations ignoring the spins and just multiply the final results by two. We also set the lattice constant $a$, the electron charge $e$, and the Planck constant $\hbar$ to unity $(e, \hbar, a=1)$ at the intermediate stages of the calculations and restore the full units only in the final results. In Eq. (1), $i W_{k}$ is the DDW order parameter with the $i d_{x^{2}-y^{2}}$ symmetry in the momentum space. For $\boldsymbol{Q}=(\pi, \pi)$, it is purely imaginary [11] and gives rise to spontaneous currents along the bonds of the square lattice, as shown in the left panel of Fig. 1. Although the presence of the spontaneous currents in the DDW state violates the microscopic time-reversal symmetry, there is no macroscopic violation. This happens because the DDW state preserves the combined symmetry of time reversal and translation by one lattice period. As a result, the staggered magnetic flux produced by the currents averages to zero on the macroscopic scale. So, one has to go further in order to explain the macroscopic time-reversal symmetry breaking revealed by the PKE measurements [9].

We propose to consider a generalization to the $d_{x y}+$ $i d_{x^{2}-y^{2}}$ density wave, where the order parameter is a combination of two density waves with different angular patterns:

$$
\left\langle\hat{c}_{\boldsymbol{k}+\boldsymbol{Q}, \alpha}^{\dagger} \hat{c}_{\boldsymbol{k}, \beta}\right\rangle \propto\left[i W_{\boldsymbol{k}}-\Delta_{\boldsymbol{k}}\right] \delta_{\alpha \beta}
$$



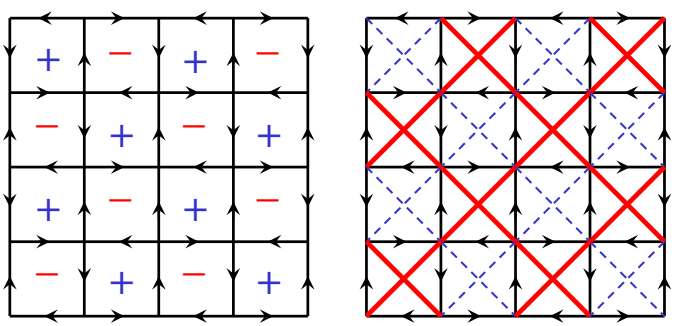

FIG. 1: Left: The real-space picture of the $i d_{x^{2}-y^{2}}$ density-wave state. Spontaneous currents are shown by the arrows. Clockwise and counterclockwise circulations are indicated by the - and the + signs, respectively. Right: The same for the $d_{x y}+i d_{x^{2}-y^{2}}$ densitywave state. Now the neighboring plaquettes have different amplitudes of the next-nearest-neighbor tunneling, indicated by the solid and dashed diagonal lines.

$$
\Delta_{k}=\Delta_{0} \sin k_{x} \sin k_{y}
$$

The real-space structure of this order parameter [11] is shown in the right panel of Fig. 1. The solid and dashed diagonal lines represent the staggered modulation of the next-nearestneighbor electron tunneling due to the $d_{x y}$ component of the density wave. This staggered modulation breaks the symmetry between the plaquettes with positive and negative circulations, so the macroscopic time-reversal symmetry is violated.

The Hartree-Fock Hamiltonian describing the mean-field $(d+i d)$-DW is

$$
\begin{gathered}
\hat{H}=\left(\begin{array}{cc}
\varepsilon_{\boldsymbol{k}}-\mu & i W_{\boldsymbol{k}}-\Delta_{\boldsymbol{k}} \\
-i W_{\boldsymbol{k}}-\Delta_{\boldsymbol{k}} & \varepsilon_{\boldsymbol{k}+\boldsymbol{Q}}-\mu
\end{array}\right), \\
\varepsilon_{\boldsymbol{k}}=-2 t\left(\cos k_{x}+\cos k_{y}\right)+4 t^{\prime} \cos k_{x} \cos k_{y},
\end{gathered}
$$

where $\varepsilon_{\boldsymbol{k}}$ is the band dispersion of the electrons, and $\mu$ is the chemical potential. The Hamiltonian (4) operates on the two-component spinor $\hat{\Psi}_{\boldsymbol{k}}=\left(\hat{c}_{\boldsymbol{k}}, \hat{c}_{\boldsymbol{k}+\boldsymbol{Q}}\right)$ and can be expanded over the Pauli matrices $\hat{\tau}$ and the unity matrix $\hat{I}$, $\hat{H}=w_{0}(\boldsymbol{k}) \hat{I}+\boldsymbol{w}(\boldsymbol{k}) \cdot \hat{\boldsymbol{\tau}}, \quad w_{0}=\frac{\varepsilon_{\boldsymbol{k}}+\varepsilon_{\boldsymbol{k}+Q}}{2}-\mu, w_{1}=$ $-\Delta_{\boldsymbol{k}}, \quad w_{2}=-W_{\boldsymbol{k}}, \quad w_{3}=\frac{\varepsilon_{\boldsymbol{k}}-\varepsilon_{\boldsymbol{k}+Q}}{2}$.

The spectrum of the Hamiltonian consists of two branches with the eigenenergies $E_{ \pm}(\boldsymbol{k})=w_{0}(\boldsymbol{k}) \pm w(\boldsymbol{k})$, where $w(\boldsymbol{k})=|\boldsymbol{w}(\boldsymbol{k})|$. For a generic set of parameters, corresponding to a non-zero hole doping in the underdoped regime, the reconstructed Fermi surface consists of two hole pockets near the $(\pi / 2, \pm \pi / 2)$ points and one electron pocket near the $(\pi, 0)$ point in the reduced Brillouin zone, as shown in Fig. 2. The Hamiltonian (4) with $t^{\prime}=0$ and $\mu=0$ was studied in Ref. [13], where it was found that this system exhibits the intrinsic quantum Hall effect without an external magnetic field. This confirms that the time-reversal symmetry is spontaneously broken in the $(d+i d)$ density-wave state.

Polar Kerr effect in the $d+i d$ density-wave state. The polar Kerr angle $\theta_{K}$ can be expressed in terms of the imaginary part of the ac Hall conductivity $\sigma_{x y}^{\prime \prime}(\Omega)$ at a frequency $\Omega[14]$,

$$
\theta_{K}=\frac{4 \pi}{n\left(n^{2}-1\right) \Omega d} \sigma_{x y}^{\prime \prime}(\Omega)
$$

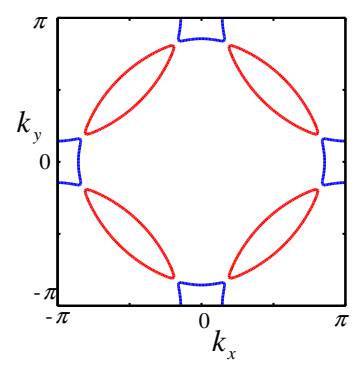

FIG. 2: The Fermi surface of the $d_{x y}+i d_{x^{2}-y^{2}}$ density-wave state, consisting of the electron and hole pockets. The hole pockets are located near $(\pi / 2, \pm \pi / 2)$, and the electron pocket near $(\pi, 0)$ in the reduced Brillouin zone. The band-structure parameters are given at the end of the paper, and $W_{0}=20 \mathrm{meV}$ is taken for this plot.

where $\sigma_{x y}$ is the Hall conductivity per 2D layer, which is related to the 3D conductivity by the interlayer distance $d$, and $n$ is the refractive index of the system. The Kerr angle was calculated for a $p_{x}+i p_{y}$ chiral superconductor [15], motivated by a non-zero PKE in $\mathrm{Sr}_{2} \mathrm{RuO}_{4}$ [16]. Eq. (6) is valid when the imaginary part of $n$ is small relative to the real part. Otherwise, there is also an additional contribution [14] from the real part $\sigma_{x y}^{\prime}(\Omega)$. For simplicity, we focus on $\sigma_{x y}^{\prime \prime}(\Omega)$, but $\sigma_{x y}^{\prime}(\Omega)$ can be also easily obtained from our calculations.

Introducing the Lagrangian density, $\mathcal{L}=i \omega_{n} \hat{I}-\hat{H}(\boldsymbol{k})$, where $\omega_{n}$ is a fermionic Matsubara frequency, we obtain the electron Green's function as a function of $\vec{k}=\left(i \omega_{n}, k_{x}, k_{y}\right)$

$$
\begin{aligned}
& G_{0}(\vec{k})=\mathcal{L}^{-1}=\frac{\left[i \omega_{n}-w_{0}(\boldsymbol{k})\right] \hat{I}+\boldsymbol{w}(\boldsymbol{k}) \cdot \hat{\boldsymbol{\tau}}}{g\left(\omega_{n}, \boldsymbol{k}\right)}, \\
& g(\vec{k})=\left[i \omega_{n}-w_{0}(\boldsymbol{k})\right]^{2}-|\boldsymbol{w}(\boldsymbol{k})|^{2}
\end{aligned}
$$

In order to calculate the Hall conductivity, we consider the electromagnetic potential $\vec{A}=\left(A_{0}, A_{x}, A_{y}\right)$, where $A_{0}$ is the scalar potential, and $\boldsymbol{A}=\left(A_{x}, A_{y}\right)$ is the vector potential. It is introduced into the Lagrangian density $\mathcal{L}$ by the standard substitution $\boldsymbol{k} \rightarrow \boldsymbol{k}-\boldsymbol{A}$ and $i \omega_{n} \rightarrow i \omega_{n}-A_{0}$, where $c$ is absorbed into $\boldsymbol{A}$. Expanding $\mathcal{L}$ to the first order in $\vec{A}$, we find the following addition to the Lagrangian density

$$
\Gamma(\vec{q}, \vec{k})=-A_{0}(\vec{q}) \hat{I}+\boldsymbol{A}(\vec{q}) \cdot \nabla_{\boldsymbol{k}}[\boldsymbol{w}(\boldsymbol{k}) \cdot \hat{\boldsymbol{\tau}}] .
$$

Here the electromagnetic potential $\vec{A}(\vec{q})$ is assumed to be a function of the Fourier variables $\vec{q}=\left(i \Omega_{m}, q_{x}, q_{y}\right)$, where $\Omega_{m}$ is a bosonic Matsubara frequency. The Lagrangian (9) operates between the fermionic spinors $\hat{\Psi}(\vec{k})$ and $\hat{\Psi}^{\dagger}(\vec{k}+\vec{q})$. Calculating the one-loop Feynman diagrams, we find the following effective action to the second order in $\Gamma$,

$$
S=\frac{1}{2} \operatorname{Tr} \sum_{\vec{k}, \vec{q}} \Gamma(\vec{q}, \vec{k}) G_{0}(\vec{k}) \Gamma(-\vec{q}, \vec{k}+\vec{q}) G_{0}(\vec{k}+\vec{q}) .
$$

Substituting Eqs. (7), (8), and (9) into Eq. (10), we write the effective action in the form

$$
S=\sum_{\vec{k}, \vec{q}} \frac{\operatorname{Tr} C_{1}}{C_{2}} .
$$


where $C_{1}$ is the product of the numerators from Eq. (7) and Eq. (9), and $C_{2}=g(\vec{k}+\vec{q}) g(\vec{k})$.

Calculating the trace over the $\hat{\tau}$ matrices in the numerator of Eq. (11), we look for the Chern-Simons-like terms, which are responsible for the Hall effect. Indeed, we find two such contributions to the effective action, $S_{H}=S_{1}+S_{2}$,

$$
\begin{aligned}
& S_{1}=i \sum_{\vec{q}} \sigma_{x y} A_{0}(\vec{q})\left[q_{x} A_{y}(-\vec{q})-q_{y} A_{x}(-\vec{q})\right] \\
& S_{2}=\sum_{\vec{q}} \frac{\Omega_{m}}{2} \sigma_{x y}\left[A_{x}(\vec{q}) A_{y}(-\vec{q})-A_{y}(\vec{q}) A_{x}(-\vec{q})\right]
\end{aligned}
$$

By taking the trace in Eq. (11), we find the following expression for the coefficient $\sigma_{x y}$ in Eqs. (12) and (13)

$$
\begin{aligned}
& \sigma_{x y}\left(\Omega_{m}\right)=\sum_{\vec{k}} \frac{B(\boldsymbol{k})}{g\left(\omega_{n}, \boldsymbol{k}\right) g\left(\omega_{n}+\Omega_{m}, \boldsymbol{k}\right)}, \\
& B(\boldsymbol{k})=-2 \boldsymbol{w} \cdot\left[\frac{\partial \boldsymbol{w}}{\partial k_{x}} \times \frac{\partial \boldsymbol{w}}{\partial k_{y}}\right],
\end{aligned}
$$

where the vector function $\boldsymbol{w}(\boldsymbol{k})$ is taken from the Hamiltonian. Indeed, the numerator in Eq. (11) is a product of four terms, each containing the $\hat{\tau}$ matrices. The Chern-Simons action (12) and (13) is obtained when these terms contribute different $\hat{\tau}$ matrices and the unity matrix to the trace. Using the formula $\operatorname{Tr}\left[\hat{\tau}_{l} \hat{\tau}_{m} \hat{\tau}_{n}\right]=2 i \epsilon_{l m n}$, where $\epsilon_{l m n}$ is the antisymmetric tensor, we arrive at Eq. (15). We also expanded the numerator of Eq. (11) to the first order in $q$ in order to obtain Eq. (12) and then took the limit $\boldsymbol{q} \rightarrow 0$, which is appropriate for the electromagnetic response of non-relativistic electrons. Substituting the expression for $\boldsymbol{w}(\boldsymbol{k})$ from the Hamiltonian into the general formula (15), we find $B(\boldsymbol{k})$ for the $d+i d$ density wave, $B(\boldsymbol{k})=4 t \Delta_{0} W_{0}\left(\sin ^{2} k_{y}+\cos ^{2} k_{y} \sin ^{2} k_{x}\right)$. Notice that $B(\boldsymbol{k})$ is proportional to the product of $\Delta_{0}$ and $W_{0}$, so the intrinsic Hall conductivity is non-zero only when both components of the $d+i d$ density wave are present. The function $B(\boldsymbol{k})$ in Eqs. (15) and above is the Berry curvature for the Hamiltonian [17].

The coefficient $\sigma_{x y}$ in Eqs. (12) and (13) is the Hall conductivity. Indeed, taking the variation $j_{x}(\vec{q})=\delta S_{H} / \delta A_{x}(-\vec{q})$, we find the Hall relation at a finite frequency

$$
j_{x}\left(\Omega_{m}\right)=\sigma_{x y}\left(\Omega_{m}\right) E_{y}\left(\Omega_{m}\right)
$$

where $\boldsymbol{E}=-i \boldsymbol{q} A_{0}-\Omega_{m} \boldsymbol{A}$ is the electric field. Now we perform summation over the fermionic Matsubara frequency $\omega_{n}$ in Eq. (14) and analytic continuation to the real bosonic frequency $i \Omega_{m} \rightarrow \Omega+i \delta$, where $\delta$ is infinitesimal. The result is

$\sigma_{x y}(\Omega)=\int_{\mathrm{RBZ}} \frac{d^{2} k}{(2 \pi)^{2}} \frac{B(\boldsymbol{k})\left\{N_{F}\left[E_{+}(\boldsymbol{k})\right]-N_{F}\left[E_{-}(\boldsymbol{k})\right]\right\}}{w(\boldsymbol{k})[\Omega+i \delta-2 w(\boldsymbol{k})][\Omega+i \delta+2 w(\boldsymbol{k})]}$,

where $N_{F}(E)$ is the Fermi occupation function for a temperature $T$, and $2 w(\boldsymbol{k})=E_{+}(\boldsymbol{k})-E_{-}(\boldsymbol{k})$ is the energy gap between the bands. The integral over $k$ is taken over the RBZ.
Let us now explore different limiting cases for Eq. (17). First we consider the de limit $\Omega=0$ at $T=0$ in the case when there are no pockets, i.e. when $E_{+}(\boldsymbol{k})>0$ and $E_{-}(\boldsymbol{k})<0$ for all $\boldsymbol{k}$. This situation takes place, e.g., when $t^{\prime}=0$ and $\mu=0$. In this case, using Eq. (15), we can write Eq. (17) as

$$
\sigma_{x y}(0)=-\frac{1}{2 \pi} \int_{\mathrm{RBZ}} \frac{d^{2} k}{4 \pi} \boldsymbol{n} \cdot\left[\frac{\partial \boldsymbol{n}}{\partial k_{x}} \times \frac{\partial \boldsymbol{n}}{\partial k_{y}}\right]
$$

where $\boldsymbol{n}(\boldsymbol{k})=\boldsymbol{w}(\boldsymbol{k}) / w(\boldsymbol{k})$ is a unit vector. The integral in Eq. (18) is an integer topological invariant, which represents the degree of mapping from the vector $k$ to the unit vector $\boldsymbol{n}$. Using Eqs. (15) and (18), we indeed find the integer quantum Hall effect per one spin component $\sigma_{x y}(0)=$ $\operatorname{sign}\left(t W_{0} \Delta_{0}\right) \frac{e^{2}}{h}$, where we restored the dimensional units. The Hall conductivity does not depend on the magnitude of the parameters $t, W_{0}$, and $\Delta_{0}$, but depends on the sign of their product.

When the pockets are present, the Fermi occupation functions in Eq. (17) exclude the pockets area from the integration over $\boldsymbol{k}$, which we represent symbolically as $\overline{\mathrm{RBZ}}$

$$
\sigma_{x y}(0)=\frac{e^{2}}{\hbar} \int_{\frac{\mathrm{RBZ}}{2 \pi)^{2}}} \frac{d^{2} k}{4 w^{3}(\boldsymbol{k})} .
$$

In this case, the Hall conductivity is no longer an integer. At a finite temperature $T$, the Fermi occupation functions in Eq. (17) further reduce the Hall conductivity.

Now we turn to the calculation of the imaginary part of the ac Hall conductivity $\sigma_{x y}^{\prime \prime}(\Omega)$. The imaginary part comes from the pole in Eq. (17) at $\Omega=2 w(\boldsymbol{k})$. This pole represents vertical interband transitions induced by the electromagnetic wave. Taking the imaginary part of (17), we find

$$
\sigma_{x y}^{\prime \prime}(\Omega)=\frac{1}{4 \pi \Omega^{2}} \frac{\int_{\mathrm{RBZ}}}{{ }_{\mathrm{RB}}} k B(\boldsymbol{k}) \delta[\Omega-2 w(\boldsymbol{k})] .
$$

The integral over $\boldsymbol{k}$ in Eq. (20) is taken along the line in the Brillouin zone where the condition $\Omega=2 w(\boldsymbol{k})$ is satisfied. Eq. (20) is valid both for $T=0$ and $T \neq 0$, as long as the condition $\Omega \gg T$ is satisfied.

Now we make a crude comparison of the theoretical result for the Kerr angle, given by Eqs. (6) and (20), with the experimental measurements [9]. We use the following representative band structure parameters: $t=300 \mathrm{meV}, t^{\prime}=90 \mathrm{meV}$, and $\mu=-260 \mathrm{meV}$. The refractive index is $n \approx 1.69$ [18], and the interlayer distance is $d=1.17 \mathrm{~nm}$. Assuming that the pseudogap originates from the ordered DDW state, we use the formula $W_{0}(x)=30\left(1-x / x_{c}\right) \mathrm{meV}$ with $x_{c}=0.175$, to model roughly how $W_{0}$ varies with the hole concentration $x$ [19]. The optical measurements were performed at the fre, quency $\hbar \Omega=0.8 \mathrm{eV}$. Taking $\Delta_{0}=\eta W_{0} / 2$, with $\eta=0.1 \%$, we plot the value of the Kerr angle $\theta_{K}$ vs. the hole doping $x$ in Fig. 3, where we have multiplied the results by 2 to account for the contributions from two spin components. The magnitude $\theta_{K}$ roughly corresponds to the experimental values of the order of $100 \mathrm{nrad}$ to $1 \mu \mathrm{rad}$ for different dopings $x$ [9]. 


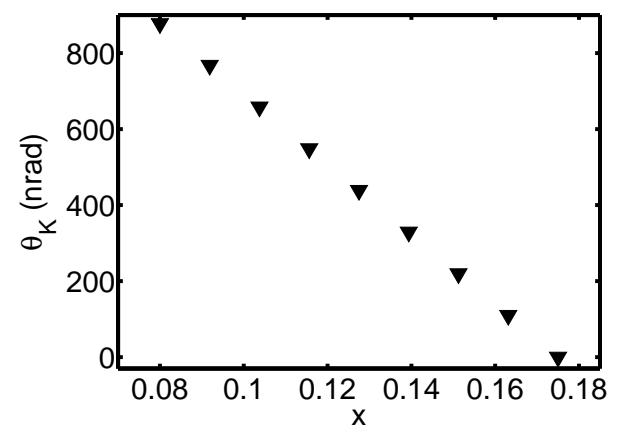

FIG. 3: The calculated Kerr angle as a function of the hole concentration $x$. We use the linear dependence $W_{0}(x)=W_{0}\left(1-x / x_{c}\right)$ to model roughly the pseudogap variation with $x$, where $x_{c}=0.175$ and $W_{0}=30 \mathrm{meV}$. Although the admixture $\Delta_{0}=0.001 W_{0} / 2$ of the $d_{x y}$ component is small, it leads to a non-zero Kerr angle of the same order of magnitude as in the experiments [9].

The intrinsic dc Hall effect predicted by Eq. (19) was not observed in the underdoped cuprates experimentally. We believe this is because of the macroscopic domains with opposite chiralities present in a sample. Their contributions to the dc Hall effect would cancel out. On the other hand, the optical measurements [9] are performed with a small laser beam within a single domain, so they give a non-zero effect.

Within our theoretical model, the PKE is possible only when both the $d_{x y}$ and $i d_{x^{2}-y^{2}}$ order parameters are present. Their transition temperatures are generally different, so the onset of the PKE corresponds to the lower of the two transition temperatures. On the other hand, the higher transition temperature may be related to the "training" or "memory" effect for the sign of the PKE [9]. The transition temperatures of the $d_{x y}$ and $i d_{x^{2}-y^{2}}$ density waves should be taken as phenomenological parameters, because we cannot reliably calculate them from the microscopic first principles.

In summary, we have developed a minimal theoretical model which can explain the macroscopic time-reversal- symmetry breaking leading to a non-zero polar Kerr effect [9] in the underdoped cuprates. By detailed calculations, we show that admixing a very small component of the $d_{x y}$ order parameter into the postulated ordered DDW state picture of the pseudogap produces a PKE comparable in magnitude to that observed in Ref. [9]. Since the necessary $d_{x y}$ admixture is very small, only of the order of $0.1 \%$, all other aspects of the YBCO phenomenology remain unaffected by our theory.

We thank Aharon Kapitulnik for sharing with us his unpublished data ([9]), and for discussion. We thank Roman Lutchyn and S. Chakravarty for interesting discussions. This work is supported by ARO-DARPA and ARO-DTO.

[1] M. R. Norman et al., Adv. Phys. 54, 715 (2005).

[2] S. A. Kivelson et al., Rev. Mod. Phys. 75, 1201 (2003).

[3] S. Chakravarty et al., Phys. Rev. B 63, 094503 (2001).

[4] S. Tewari et al., Phys. Rev. B 70, 014514 (2004).

[5] S. Chakravarty et al., Nature 428, 53 (2004).

[6] N. Doiron-Leyraud et al., Nature 447, 565 (2007); A. F. Bangura et al., Phys. Rev. Lett. 100, 047004 (2008).

[7] S. Chakravarty and H.-Y. Kee, arXiv:0710.0608 (2007).

[8] S. Chakravarty, C. Nayak, and S. Tewari, Phys. Rev B 68, 100504 (2003).

[9] J. Xia et al., arXiv:0711.2494

[10] J. E. Sonier et al., Science 292, 1692 (2001).

[11] C. Nayak, Phys. Rev. B 62, 4880 (2000).

[12] G. M. Luke et al., Nature 394, 558 (1998); Y. Aoki et al., Phys. Rev. Lett. 91, 067003 (2003); Y. Kasahara et al., Phys. Rev. Lett. 99, 116402 (2007).

[13] V. M. Yakovenko, Phys. Rev. Lett. 65, 251 (1990).

[14] R. M. White and T. H. Geballe, Long Range Order in Solids (Academic Press, New York, 1979), pp. 317, 321.

[15] V. M. Yakovenko, Phys. Rev. Lett. 98, 087003 (2007).

[16] J. Xia et al., Phys. Rev. Lett. 97, 167002 (2006).

[17] M. V. Berry, Proc. R. Soc. London A 392, 92 (2003).

[18] H. Kezuka et al., Physica C 185, 999 (1991).

[19] J. W. Loram et al., J. Phys. Chem. Solids 62, 59 (2001). 